\title{
AC Electrical Conductivity and Dielectric Properties of Perovskite $(\mathrm{Pb}, \mathrm{Ca}) \mathrm{TiO}_{3}$ Ceramic
}

\author{
H.M. El-MaLlah* \\ Department of Physics \& Mathematical Engineering, Faculty of Engineering, Port-Said University \\ 42523 Port-Said, Egypt
}

(Received July 22, 2011; in final form April 14, 2012)

\begin{abstract}
$\mathrm{Pb}_{1-x} \mathrm{Ca}_{x} \mathrm{TiO}_{3}$ perovskite crystalline structure with $x=0,0.2,0.6,0.7$, and 0.8 were prepared by mixture method. The ac conductivity and dielectric properties of the studied bulk compositions have been investigated in the frequency range $1 \times 10^{3}-5 \times 10^{6} \mathrm{~Hz}$ and temperature range $303-473 \mathrm{~K}$. The experimental results indicate that the ac conductivity $\sigma_{\mathrm{ac}}(\omega)$, dielectric constant $\varepsilon^{\prime}$ and dielectric loss $\varepsilon^{\prime \prime}$ depend on the temperature and frequency. The ac conductivity was found to obey the power law $\omega^{S}$ with the frequency exponent $S>1$ decreasing with increasing temperature. The present results are compared to the principal theories that describe the universal dielectric response behavior. Values of dielectric constant $\varepsilon^{\prime}$ and dielectric loss $\varepsilon^{\prime \prime}$ were found to be temperature and frequency dependent and the maximum barrier height $W_{\mathrm{m}}$ is calculated.
\end{abstract}

PACS: 71.45.Gm, 72.20.-i, 72.80.-r

\section{Introduction}

Dielectric and ferroelectric materials have been the subject of renewed attention. These materials applications have been widely employed in various industrial devices such as dynamic access memory, microwave filters, voltage controlled oscillators and telecommunication technologies [1-4]. Conventional dielectrics and ferroelectrics are mainly lead-based ceramic materials, which have been considered to be a serious threat to the environment [5]. Many attentions have been paid to improve the electric and dielectric properties of $\mathrm{ABO}_{3}$ perovskite via partial substitution of either A-ions ( $\mathrm{Pb}, \mathrm{Ca}, \mathrm{Ba}$ or $\mathrm{Sr}$ site doping) or B-ions (Ti site doping) [6]. So far, many new dielectric materials have been developed in recent years due to their interesting properties [7-10].

Ac conductivity measurements have been widely used to investigate the nature of ionic motion in ionically conducting materials since it is assumed that they are responsible for this type of conduction. It is well established that the electrical behavior of complex ionically conducting materials as a function of frequency shows two regions of behavior named the universal dielectric response (UDR) [11] and nearly constant loss (NCL) in which the dielectric loss $\varepsilon^{\prime \prime}$ is nearly constant [12-14]. The dependence of the ac conductivity $\sigma_{\mathrm{ac}}(\omega)$ on the frequency is found to obey the form

$$
\sigma_{\mathrm{ac}}(\omega)=A \omega^{S}
$$

where $\sigma_{\mathrm{ac}}(\omega)$ is the ac conductivity, $\omega$ - the angular frequency, $A-$ constant and $S$ - the frequency exponent. Such behavior was first reported by Jonscher [15, 16] for a wide variety of materials and defined as the UDR. A wide variety of theoretical approaches have been used to clarify the UDR behavior, in general involving hopping of

* e-mail: hanaaelmallah53@hotmail.com carrier ions with appropriate relaxation of surrounding ions which occurs in the crystalline or glassy materials. In the NCL model, at high frequency and low temperature the frequency exponent $S$ tends to the value of 1 . (Actually, it is often found to go slightly higher to a value $\approx 1.0-1.12$.) A value of $S=1$ corresponds to $\varepsilon^{\prime \prime}$ equal constant value, independent of frequency and varies only slowly with temperature i.e. it is not activated. This behavior can be observed down to cryogenic temperatures $[12,13]$.

This paper concerns with the $\mathrm{Pb}_{1-x} \mathrm{Ca}_{x} \mathrm{TiO}_{3}$ (PCT), which represents a member of perovskite materials with the varying $\mathrm{Ca}$ concentration as $x=0,0.2,0.6,0.7$, and 0.8 . The objective of this paper is to study the influence of the varying $\mathrm{Ca}$ concentration on the ac conductivity and dielectric properties with a view to understanding the conduction mechanism involved. The frequency and temperature dependences of the ac conductivity, dielectric constant, and dielectric loss have been also studied through the temperature range $303-473 \mathrm{~K}$ and frequency range $1 \times 10^{3}-5 \times 10^{6} \mathrm{~Hz}$.

\section{Experimental details}

Five bulk compositions of the $\mathrm{Pb}_{1-x} \mathrm{Ca}_{x} \mathrm{TiO}_{3}$ system with nominal compositions $0,0.2,0.6,0.7$, and 0.8 were prepared in the form of a disc with $1.3 \mathrm{~cm}$ in diameter and uniform thickness. Details for sample synthesis can be found in previous work [17]. These samples were covered on the opposite surfaces with thin layer of silver paste film to obtain a good contact and were mounted between two copper electrodes in a special holder. The sample holder was inserted in a small furnace and the temperature was measured to better than $\pm 1{ }^{\circ} \mathrm{C}$ with calibrated chromel-alumel thermocouple which was set up near the sample. Ac conductivity and dielectric properties of the $\mathrm{Pb}_{1-x} \mathrm{Ca}_{x} \mathrm{TiO}_{3}$ system with nominal compositions $0,0.2,0.6,0.7$, and 0.8 were measured using 
Hioki 3532 LCR meter. The measurements were carried out in the frequency range $1 \times 10^{3}-5 \times 10^{6} \mathrm{~Hz}$ under a controlled temperature that varied from 303 to $473 \mathrm{~K}$.

A programmable automatic 3532 LCR meter was used to measure the sample impedance $Z$, the sample capacitance $C_{p}$ and the loss tangent $\tan \delta$ directly. All values of the capacitance $C_{p}$ which taken from the screen of the bridge were parallel with the resistance $R$.

The total conductivity $\sigma_{\mathrm{t}}(\omega)$ was calculated from the equation

$$
\sigma_{\mathrm{t}}(\omega)=L / Z a,
$$

where $L$ is the sample thickness and $a$ - the sample cross-sectional area, ac conductivity $\sigma_{\mathrm{ac}}(\omega)$ was calculated using the relation

$$
\sigma_{\mathrm{ac}}(\omega)=\sigma_{\mathrm{t}}(\omega)-\sigma_{\mathrm{d}}(0),
$$

where $\sigma_{\mathrm{d}}(0)$ is termed as the dc conductivity.

The dielectric constant was calculated using the relation

$$
\varepsilon^{\prime}=C_{p} L / a \varepsilon_{0},
$$

where $\varepsilon_{0}$ is the free space permittivity.

The dielectric loss $\varepsilon^{\prime \prime}$ was calculated from the relation

$$
\varepsilon^{\prime \prime}=\varepsilon^{\prime} \tan \delta
$$

where $\delta=90^{\circ}-\Phi, \Phi$ is the phase angle which has been measured using the same bridge. The values of the impedance $Z$, the capacitance $C_{p}$ and the loss tangent $\tan \delta$ were measured in the frequency range $1 \times 10^{3}-5 \times$ $10^{6} \mathrm{~Hz}$ and temperature range $303-473 \mathrm{~K}$.

\section{Results and discussion}

X-ray diffraction patterns obtained for the $\mathrm{Pb}_{1-x} \mathrm{Ca}_{x} \mathrm{TiO}_{3}$ with $x=0,0.2,0.6,0.7$, and 0.8 show that there are no traces of other compounds and the line widths indicated that the products were homogeneous, thus all the compositions are in good quality with the perovskite structure.

\subsection{Frequency and temperature dependence of ac conductivity}

The variation of $\sigma_{\mathrm{ac}}(\omega)$ with frequency in the range $1 \times 10^{3}-5 \times 10^{6} \mathrm{~Hz}$ for the investigated compositions (with similarly the same thickness) was studied through the temperature range $303-448 \mathrm{~K}$. The obtained results are plotted as $\ln \sigma_{\mathrm{ac}}(\omega)$ versus $\ln \omega$ at different elevated temperature values according to Eq. (1). Figure 1a, b shows the frequency dependence for the compositions $\mathrm{PbTiO}_{3}$ and $\mathrm{Pb}_{0.2} \mathrm{Ca}_{0.8} \mathrm{TiO}_{3}$ as examples. It is clear from this figure that $\sigma_{\mathrm{ac}}(\omega)$ increases linearly with increasing frequency. The same behavior of the frequency dependence of $\sigma_{\mathrm{ac}}(\omega)$ was obtained for all other compositions. Values of the frequency exponent $S$ were calculated from the slope of the straight lines of the data in Fig. 1a, b. Values of the frequency exponent $S$ was plotted as a function of temperature as shown in Fig. 2 for all the studied compositions. It is clear from this figure that $S$ decreases with increasing temperature.

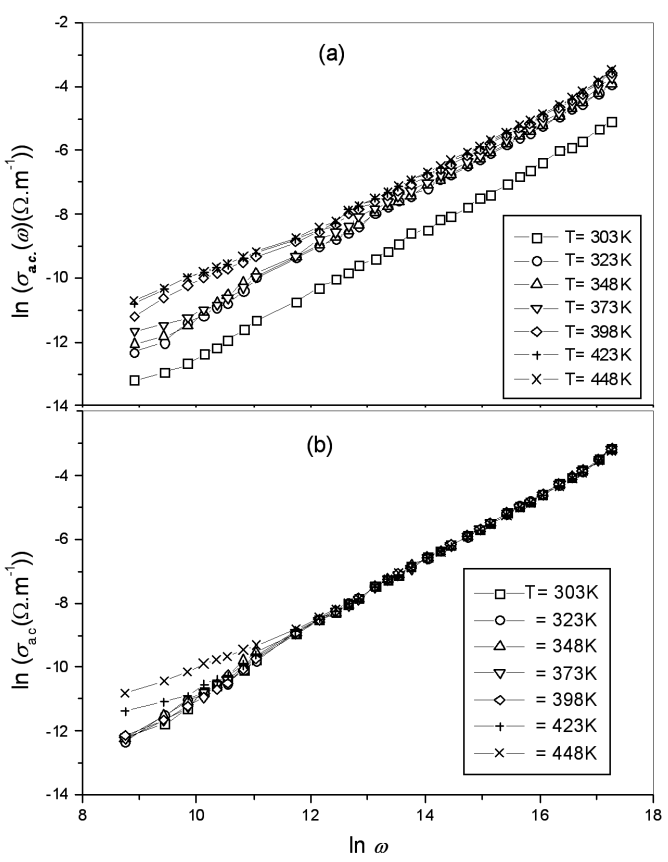

Fig. 1. Frequency dependence of $\sigma_{\mathrm{ac}}(\omega)$ for (a) $\mathrm{PbTiO}_{3}$, (b) $\mathrm{Pb}_{0.2} \mathrm{Ca}_{0.8} \mathrm{TiO}_{3}$ at different temperatures.

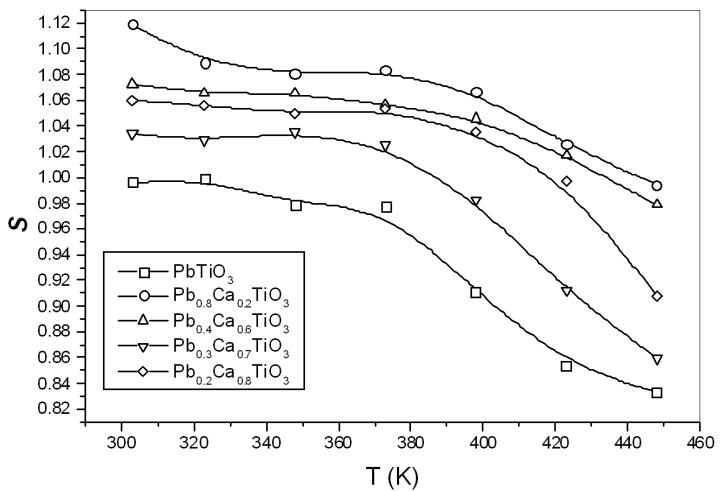

Fig. 2. Temperature dependence of the frequency exponent $S$ for PCT.

The temperature dependence of $S$ gives information to specify the suitable mechanism involved for ac conductivity. This behavior of ac conductivity is analogous to that observed in ionic glasses [15]. It is reported that the ac conductivity of highly disordered materials including amorphous semiconductors and ionic conducting glasses is obeying Eq. (1). This equation is known as the UDR which has been described by Jonscher [15]. This behavior was noted originally for amorphous semiconductors and glasses [14]. In most cases, $S$ is found to be between 0.6 and 1 for ionic conducting materials [18]. The value of $S=1$ is regarded theoretically as the limiting value. The experimental values of $S$ obtained for all compositions are illustrated in Fig. 2. The observed values of the exponent $S$ are somehow frequency dependent. 
It decreases with increasing temperature from 0.996 to 0.854 for the composition at $x=0$ and from 1.06 to 0.908 for the composition at $x=0.8$ as an example. This can be attributed to hoping conduction of mobile charge carriers (ions) over barrier between two sites, which is similar to that observed in amorphous semiconductors and glasses $[13,18]$. The case of $S>1$ has been reported in a few compositions of crystalline, semiconductors and glasses $[13,19,20]$. The motion of mobile charge carriers from site to site with quantum mechanic tunneling between asymmetric double-well potentials was proposed for that case [21]. The change of $S$ with the temperature corresponds to a thermally activated process. The high value of $S$ can be explained as follows. The doping of $\mathrm{Ca}^{2+}$ in $\mathrm{PbTiO}_{3}$ leads to the replacement of $\mathrm{Pb}^{2+}$ in its site by $\mathrm{Ca}^{2+}$ which are of the same charge. Some of $\mathrm{Ca}^{2+}$ may be located at $\mathrm{Ti}^{4+}$ sites, and the next neighbor oxygen can be vacant $\mathrm{V}_{o}$ and forming a $\mathrm{Ca}^{2+}-\mathrm{V}_{\mathrm{o}}$ neutral center which relax the hopping of conduction ions. Thus the addition of $\mathrm{Ca}^{2+}$ leads to decrease the number of $\mathrm{Pb}^{2+}-\mathrm{V}_{\mathrm{o}}$ forming neutral centers, and consequently the ac conductivity $\sigma_{\mathrm{ac}}(\omega)$ increases [22].

The temperature dependence of $\sigma_{\mathrm{ac}}(\omega)$, at different frequencies for the $\mathrm{Pb}_{1-x} \mathrm{Ca}_{x} \mathrm{TiO}_{3}$ system was measured in the range of $303-448 \mathrm{~K}$. The results obtained are shown in Fig. 3a, b where $\ln \sigma_{\mathrm{ac}}(\omega)$ are plotted as a function of $1000 / T$. It is clear from Fig. 3a that $\sigma_{\mathrm{ac}}(\omega)$ shows a frequency and temperature dependence over the whole ranges for $x=0.2$ as an example and at lower values of frequency $\left(1 \times 10^{3}-2 \times 10^{4} \mathrm{~Hz}\right)$ for the concentration $x=0.7$ as an example (Fig. $3 \mathrm{~b}$ ). The same behavior of the temperature dependence of $\sigma_{\mathrm{ac}}(\omega)$ was obtained for the concentration $x=0$ and for the concentration $x=0.6$ and 0.8 , respectively. These figures show that the relation $\sigma_{\mathrm{ac}}(\omega)=f(1 / T)$ is linear with two slopes which suggests two different regimes with different activation energies, one with weak temperature dependence and the other with strong temperature dependence. Accordingly the ac component of the frequency dependent conductivity $\sigma_{\mathrm{ac}}(\omega)$ can be expressed as the sum of two different conduction mechanisms

$$
\sigma_{\mathrm{ac}}(\omega)=\sigma_{F}+\sigma_{S} .
$$

Here $\sigma_{F}$ represents the relativity weak temperature dependence, which has been interpreted as being due to hopping of charge carriers and $\sigma_{S}$ represents the strong temperature dependence component and is obtained by subtracting $\sigma_{F}$ from $\sigma_{\text {ac }}(\omega)$. This behavior is similar to that obtained in amorphous semiconductors and glasses [23]. It is also observed from Fig. 3b that $\sigma_{\mathrm{ac}}(\omega)$ increases linearly at higher values of frequency range $\left(2 \times 10^{4}-5 \times 10^{6} \mathrm{~Hz}\right)$. The ac conductivity at higher values of frequency shows weakly temperature dependence. The value of the activation energy $\Delta E_{a}(\omega)$ is calculated at different frequencies from the slope of lines in Fig. $3 \mathrm{~b}$ using the well known equation

$$
\sigma_{\text {ac }}(\omega)=\sigma_{0} \exp \left(-\Delta E_{a}(\omega) / k_{\mathrm{B}} T\right),
$$

where $\sigma_{0}$ is a constant, $\Delta E_{a}$ - the activation energy for

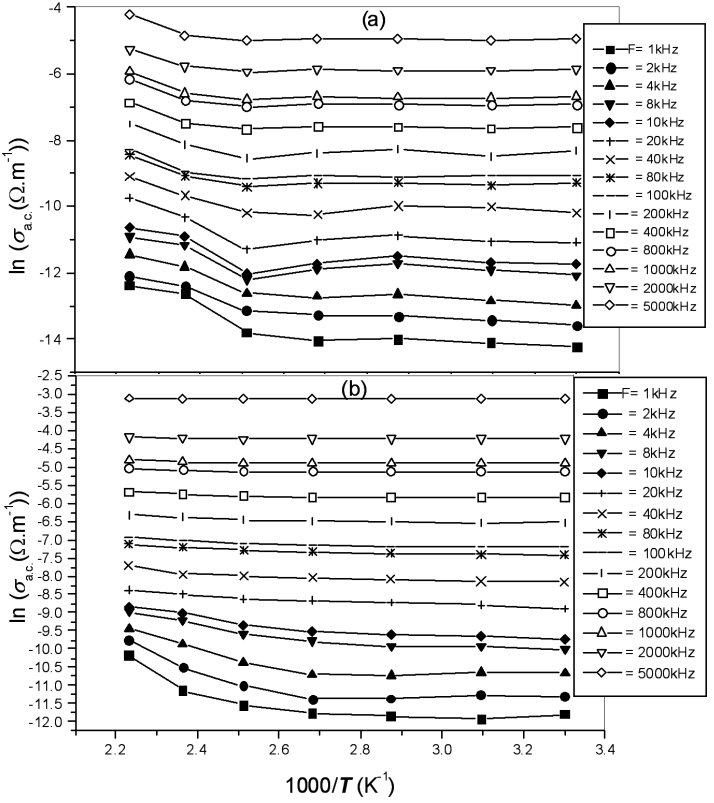

Fig. 3. Temperature dependence of $\sigma_{\mathrm{ac}}(\omega)$ for (a) $\mathrm{Pb}_{0.8} \mathrm{Ca}_{0.2} \mathrm{TiO}_{3}$ and (b) $\mathrm{Pb}_{0.3} \mathrm{Ca}_{0.7} \mathrm{TiO}_{3}$ at different frequencies.

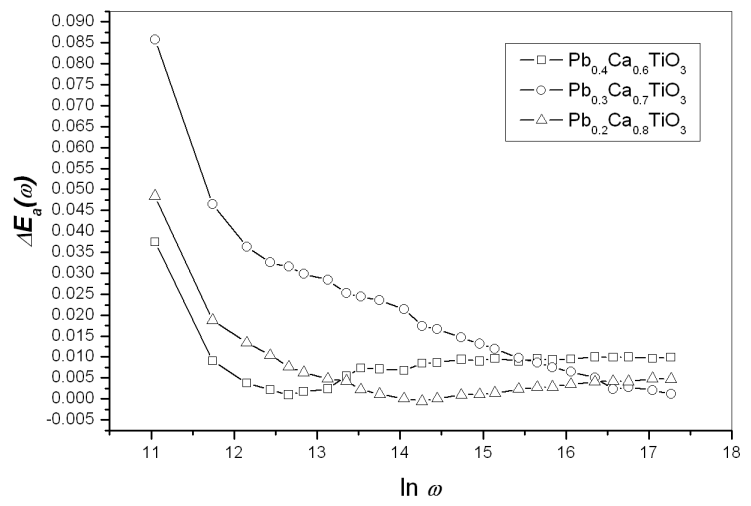

Fig. 4. Frequency dependence of activation energy $\Delta E_{a}(\omega)$ for PCT

conduction, $k_{\mathrm{B}}$ - the Boltzmann constant and $T$ - the absolute temperature.

Figure 4 shows the frequency dependence of activation energy $\Delta E_{a}(\omega)$ for compositions with $x=0.6,0.7$, and 0.8. It is clear that $\Delta E_{a}(\omega)$ decreases with increasing frequency which is similar to that obtained for other amorphous, glass, crystalline materials $[13,20]$. The smaller values of $\Delta E_{a}(\omega)$ and the increase of $\sigma_{\mathrm{ac}}(\omega)$ with increasing frequency confirm that the hopping conduction is the dominant mechanism.

\subsection{Frequency and temperature dependence of the dielectric constant}

Dielectric analysis measures the electric properties of a material as a function of frequency and temperature. 


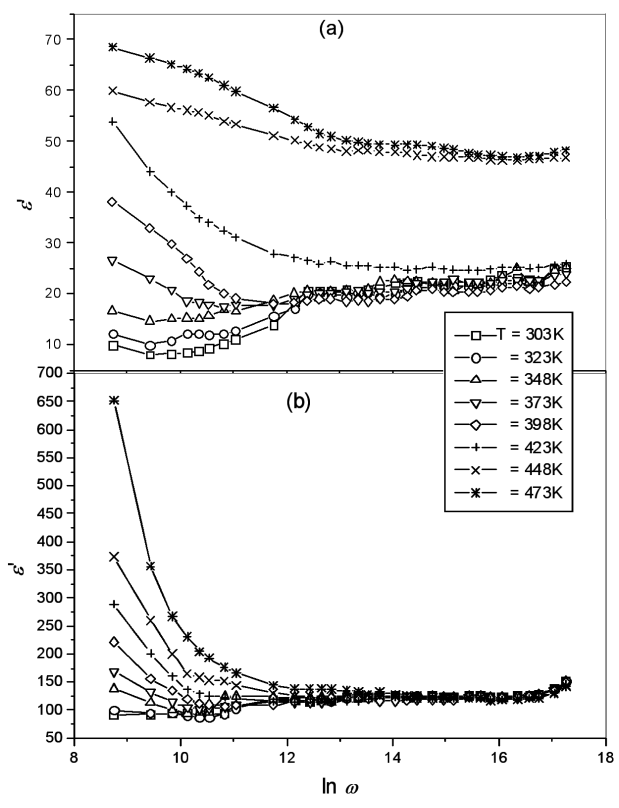

Fig. 5. Frequency dependence of dielectric constant $\varepsilon^{\prime}$ for (a) $\mathrm{Pb}_{0.8} \mathrm{Ca}_{0.2} \mathrm{TiO}_{3}$ and (b) $\mathrm{Pb}_{0.2} \mathrm{Ca}_{0.8} \mathrm{TiO}_{3}$ at different temperatures.

This analysis measures two fundamental electrical characteristic of materials: (1) the capacitive (insulating) nature, which represents its ability to store electric charge. (2) The conductive nature, which represents its ability to transfer electronic charge. Through this analysis, the dielectric constant $\varepsilon^{\prime}$ and dielectric loss $\varepsilon^{\prime \prime}$ of a material can be determined.

Figure 5a, b shows the frequency dependence of dielectric constant $\varepsilon^{\prime}$ at different temperature for the compositions $\mathrm{Pb}_{0.8} \mathrm{Ca}_{0.2} \mathrm{TiO}_{3}$ and $\mathrm{Pb}_{0.2} \mathrm{Ca}_{0.8} \mathrm{TiO}_{3}$ as examples. The same behavior of dielectric constant was obtained for other studied compositions. It is clear from this figure that $\varepsilon^{\prime}$ decreases with frequency and increases with temperature. The decrease of $\varepsilon^{\prime}$ with frequency can be attributed to the fact that at low frequencies $\varepsilon^{\prime}$ for polar material is explained by the contribution of multicomponent of polarizibility, deformational (electronic and ionic) and relaxation (orientational and interfacial) polarization.

First, electronic polarization arises from the displacement of the valence electrons relative to the positive nucleus. This type of polarization takes place at frequencies up to $10^{16} \mathrm{~Hz}$.

Second type is ionic polarization, occurs due to the displacement of negative and positive ions with respect to each other. The maximum frequency of ionic polarization is $10^{13} \mathrm{~Hz}$.

Third, dipolar polarization occurs if the material contains molecules, with permanent electric dipole moment that can change orientation into the direction of the applied electric field. The dipolar polarization takes place at frequencies up to $10^{10} \mathrm{~Hz}$.

The final one is the space charge polarization which occurs due to impedance mobile charge carriers by in- terfaces. Space charge polarization typically occurs at frequency range from 1 to $10^{3} \mathrm{~Hz}$. The total polarization of the dielectric material can be given as the sum of these four types of polarization [24]. The obtained results in the present study may be referred to the total polarization.

When the frequency is increased, the orientational polarization decreases since it takes more time than electronic and ionic polarization. This decreases the value of dielectric constant $\varepsilon^{\prime}$ reaching a constant value at higher frequency correspondingly to interfacial polarization.

Figure 5a, b shows the temperature dependence of the dielectric constant $\varepsilon^{\prime}$ for the compositions $\mathrm{Pb}_{0.8} \mathrm{Ca}_{0.2} \mathrm{TiO}_{3}$ and $\mathrm{Pb}_{0.2} \mathrm{Ca}_{0.8} \mathrm{TiO}_{3}$ as examples. It is clear from this figure that $\varepsilon^{\prime}$ increases with temperature. The increase of $\varepsilon^{\prime}$ with temperature can be attributed to the fact that the orientional polarization is connected with the thermal motion of molecules, so dipoles cannot orient themselves at low temperatures. When the temperature is increased the orientation of dipole is facilitated and this increases the value of orientational polarization, which leads to increase the dielectric constant $\varepsilon^{\prime}$ with temperature. The increase in $\varepsilon^{\prime}$ is also due to the increase in the degree of crystallinity [25-27].

\subsection{Frequency and temperature dependence of the dielectric loss}

The variation of the dielectric loss $\varepsilon^{\prime \prime}$ with the frequency at different temperatures is shown in Fig. 6a, b for the compositions $\mathrm{Pb}_{0.8} \mathrm{Ca}_{0.2} \mathrm{TiO}_{3}$ and $\mathrm{Pb}_{0.2} \mathrm{Ca}_{0.8} \mathrm{TiO}_{3}$ as examples. Similar behavior of dielectric loss has been observed for the compositions $x=0,0.6,0.7$. The magnitude of the dielectric loss increases with increase in temperature. Owing to the relaxation studies made by Stevels [28], the dielectric loss can be divided into three parts: conduction losses, dipole losses, and vibrational losses. The loss that was attributed to conduction presumably involves the migration of ions over large distances. This motion is the same as that occurring under direct current conductions. The ions jump over the highest potential in the network. As the ions move, they give some of their energy to the lattice as heat and the amount of heat lost per cycle is proportional to $\sigma_{\mathrm{ac}}(\omega) / \omega$. At low temperature conduction losses have minimum value. As the temperature increases $\sigma_{\mathrm{ac}}(\omega)$ increases, so the conduction losses increase. This leads to increase of the value of $\varepsilon^{\prime \prime}$ with temperature. The increase of the dielectric loss at low frequency is due to dipole polarization.

It is clear from Fig. 6a, b that $\varepsilon^{\prime \prime}$ decreases with increasing frequency through the studied range. The decrease of $\varepsilon^{\prime \prime}$ with frequency may be attributed to the fact that the migration of ions is the main source of the dielectric loss $\varepsilon^{\prime \prime}$ at low frequencies. Accordingly, $\varepsilon^{\prime \prime}$ at low and moderate frequency is characterized by high values of $\varepsilon^{\prime \prime}$ due to the contribution of ion jump and conduction loss of ion migration, in addition to the ion polarization. But, at higher frequency values the ion vibrations may be the only source of dielectric loss so $\varepsilon^{\prime \prime}$ decreases at 


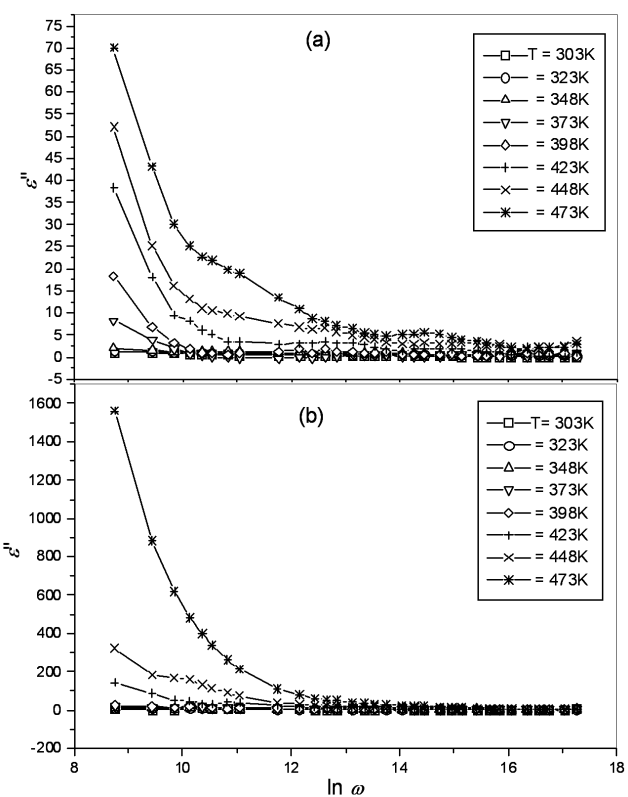

Fig. 6. Frequency dependence of dielectric loss $\varepsilon^{\prime \prime}$ for (a) $\mathrm{Pb}_{0.8} \mathrm{Ca}_{0.2} \mathrm{TiO}_{3}$ and (b) $\mathrm{Pb}_{0.2} \mathrm{Ca}_{0.8} \mathrm{TiO}_{3}$ at different temperatures.

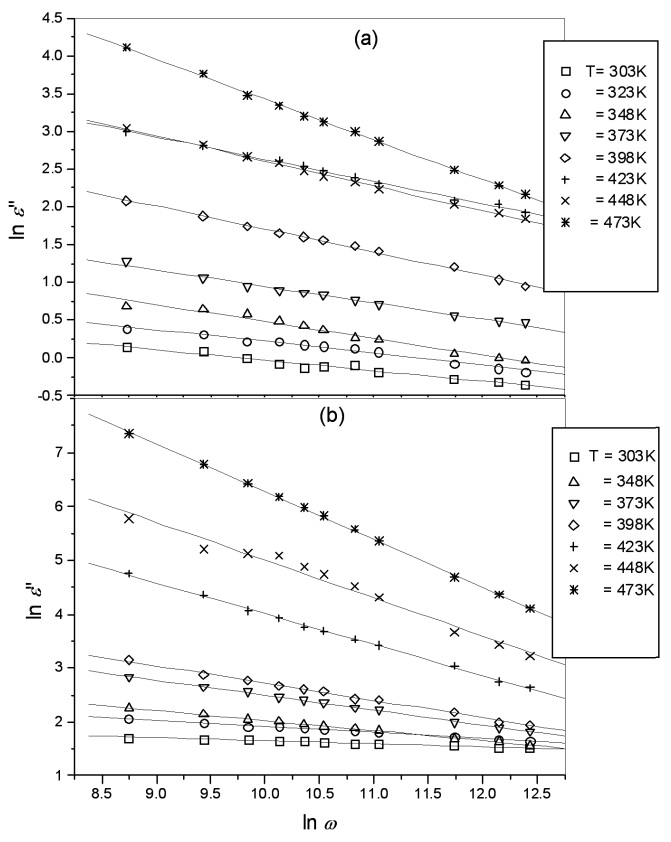

Fig. 7. $\ln \varepsilon^{\prime \prime}$ versus $\ln \omega$ for (a) $\mathrm{Pb}_{0.8} \mathrm{Ca}_{0.2} \mathrm{TiO}_{3}$ and (b) $\mathrm{Pb}_{0.2} \mathrm{Ca}_{0.8} \mathrm{TiO}_{3}$ at different temperatures.

higher values of frequency. The obtained results of $\varepsilon^{\prime \prime}$ can be analyzed according to the theory of hopping of charge carriers over a potential barrier between charged defects $[29,30]$. Based on this model the dielectric loss $\varepsilon^{\prime \prime}$ can be expressed by the following relation:

$$
\varepsilon^{\prime \prime}=A \omega^{m},
$$

where

$$
m=-4 k_{\mathrm{B}} T / W_{\mathrm{m}},
$$

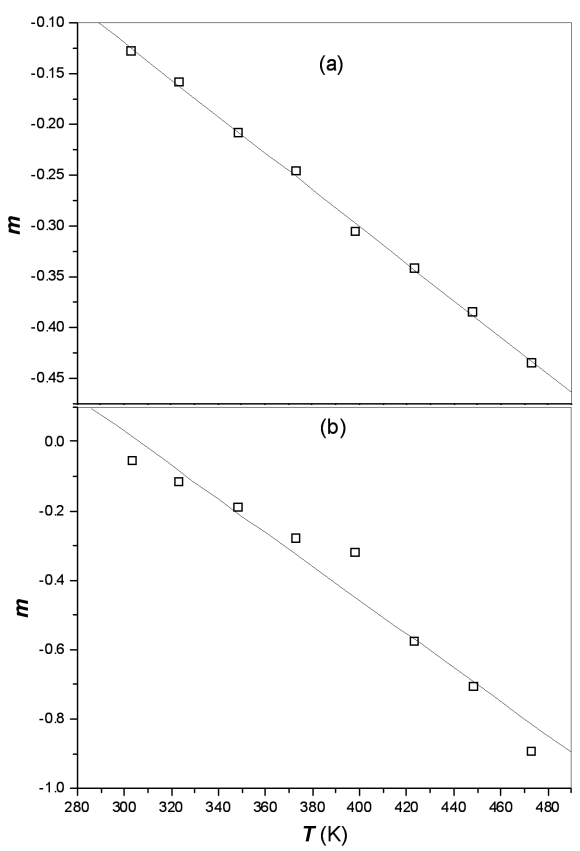

Fig. 8. Temperature dependence of the power parameter $m$ for (a) $\mathrm{Pb}_{0.8} \mathrm{Ca}_{0.2} \mathrm{TiO}_{3}$ and (b) $\mathrm{Pb}_{0.2} \mathrm{Ca}_{0.8} \mathrm{TiO}_{3}$.

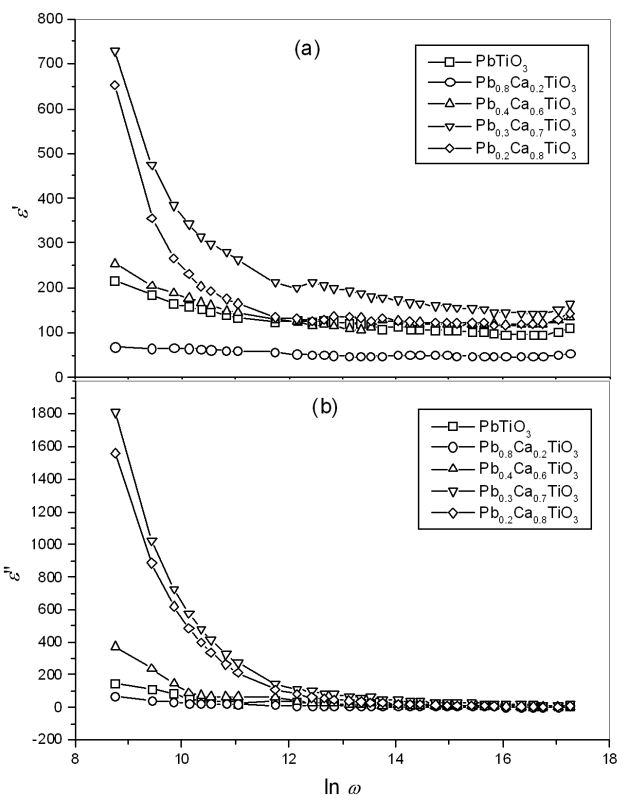

Fig. 9. Frequency dependence of dielectric constant $\varepsilon^{\prime}$ (a) and dielectric loss $\varepsilon^{\prime \prime}$ (b) at $473 \mathrm{~K}$ for PCT.

where $A$ is a constant, $m$ is the frequency power parameter and $W_{\mathrm{m}}$ is the maximum barrier height. This study of $\varepsilon^{\prime \prime}$ enables us to relate the power $m$ to the temperature of measurements. According to Eq. (8), the obtained results can be plotted as $\ln \varepsilon^{\prime \prime}$ versus $\ln \omega(\omega=2 \pi F)$ according to the Giuntini equation [31]. Figure 8a, b confirms this behavior for all studied compositions. Values of power parameter $m$ calculated from the slope of the obtained straight lines of Fig. 7a, b. Figure 8a, b shows the temperature dependence of the power param- 
eter $m$ which decreases linearly with temperature. From the slope of these straight lines and Eq. (9), the values of $W_{\mathrm{m}}$ were calculated for the studied compositions and listed in Table.

TABLE

The obtained value of the maximum barrier height $W_{\mathrm{m}}$ for all compositions.

\begin{tabular}{c|c}
\hline \hline Compositions & $W_{\mathrm{m}}[\mathrm{eV}]$ \\
\hline $\mathrm{PbTiO}_{3}$ & 0.114 \\
$\mathrm{~Pb}_{0.8} \mathrm{Ca}_{0.2} \mathrm{TiO}_{3}$ & 0.100 \\
$\mathrm{~Pb}_{0.4} \mathrm{Ca}_{0.6} \mathrm{TiO}_{3}$ & 0.091 \\
$\mathrm{~Pb}_{0.3} \mathrm{Ca}_{0.7} \mathrm{TiO}_{3}$ & 0.075 \\
$\mathrm{~Pb}_{0.2} \mathrm{Ca}_{0.8} \mathrm{TiO}_{3}$ & 0.071
\end{tabular}

Figure 9a, b shows the variation of $\varepsilon^{\prime}$ and $\varepsilon^{\prime \prime}$ for the studied compositions at $473 \mathrm{~K}$. It is clear that $\varepsilon^{\prime}$ and $\varepsilon^{\prime \prime}$ increase with increasing $\mathrm{Ca}$ addition which is in agreement with the obtained results of $\sigma_{\mathrm{ac}}(\omega)$ as mentioned above.

\section{Conclusion}

Ac conductivity and dielectric properties of $\mathrm{Pb}_{1-x} \mathrm{Ca}_{x} \mathrm{TiO}_{3}$ perovskite crystalline compositions with $x=0,0.2,0.6,0.7$, and 0.8 were studied at the frequency range $1 \times 10^{3}-5 \times 10^{6} \mathrm{~Hz}$ and temperature range $303-473 \mathrm{~K}$. The angular frequency $\omega$ dependence of ac conductivity $\sigma_{\mathrm{ac}}(\omega)$ is found to follow Jonscher UDR. The decreases of $S$ with increasing temperature are similar to those observed in amorphous semiconductors, Chalcogenide glasses and crystalline materials. However an appreciable difference in the temperature dependence of $S$ is observed. The frequency and temperature dependence of ac conductivity, the small values of ac activation energy $\Delta E_{a}(\omega)$, suggest that the hopping conduction is operating mechanism. In addition, the decreasing temperature dependence of the frequency exponent $S$ points out to the correlated barrier hopping (CBH) mechanism for ac conduction.

Both the dielectric constant $\varepsilon^{\prime}$ and dielectric loss $\varepsilon^{\prime \prime}$ increase with temperature and decrease with frequency through the studied ranges. The dielectric constant may be attributed to orientation and space charge polarization respectively, where as the temperature dependence of the dielectric loss is associated with the conduction loss. Values of the maximum barrier height $W_{\mathrm{m}}$ are in agreement with the theory of hopping of charge carriers over a potential barrier between charged defects as that occurs in other dielectric materials. The increase of $\mathrm{Ca}$ content in the investigated system leads to increase the ac conductivity, the dielectric constant, the dielectric loss and decreases the maximum barrier height.

\section{References}

[1] A. Ioachim, M.I. Toacsan, M.G. Banciu, L. Nedelcu, F. Vasiliu, H.V. Alexandru, C. Berbecaru, G. Stoica, Prog. Solid State Chem. 35, 513 (2007).

[2] Y. Ota, K.I. Kakimoto, H. Ohsato, T. Okawa, J. Eur. Ceram. Soc. 24, 1755 (2004).
[3] Y.C. Chen, P.S. Cheng, C.F. Yang, W.C. Tzou, Ceram. Int. 27, 809 (2001)

[4] H. Zhou, H. Wang, Y. Chen, K. Li, X. Yao, Mater. Chem. Phys. 113, 1 (2009).

[5] R. Zuo, X. Fang, C. Ye, Appl. Phys. Lett. 90, 092904 (2007).

[6] J. Qi, Z. Gui, W. Li, Y. Wang, Y. Wu, L. Li, Mater Lett. 56, 507 (2002)

[7] L.P. Curecheriu, L. Mitoseriu, A. Ianculescu, J. Alloys Comp. 482, 1 (2009).

[8] A.K. Nath, K.C. Singh, R. Laishram, O.P. Thakur, Mater. Sci. Eng. B 172, 151 (2010).

[9] G. Aldica, M. Cernea, R. Radu, R. Trusca, J. Alloys Comp. 505, 273 (2010).

[10] V.V. Shvartsman, W. Kleemann, J. Dec, J. Appl. Phys. 99, 124111 (2006).

[11] P. Maass, M. Meyer, A. Bunde, Phys. Rev. B 51, 8164 (1995)

[12] A.R. Long, Adv. Phys. 31, 553 (1982).

[13] S.R. Elliott, Adv. Phys. 36, 135 (1987); Solid State Ion. 70-71, 27 (1994).

[14] D.L. Sidebottom, P.F. Green, R.K. Brow, J. Non-Cryst. Solids 203, 300 (1996).

[15] A.K. Jonscher, Nature 267, 673 (1977).

[16] A.K. Jonscher, Dielectric Relaxation in Solids, Chelsea Dielectrics Press, London 1983.

[17] H.M. El-Mallah, J. Mater. Sci. 39, 1711 (2004).

[18] W.K. Lee, J.F. Liu, A.S. Nowick, Phys. Rev. Lett. 67, 1559 (1994)

[19] R.H. Chen, R.J. Wang, T.M. Chen, C.S. Shern, J. Phys. Chem. Solids 61, 519 (2000).

[20] H.M. El-Mallah, N.A. Hegab, J. Mater. Sci. 42, 336 (2007).

[21] K.S. Gilroy, W.A. Phillips, Philos. Mag. B 43, 735 (1981).

[22] J.G. Bednorz, K.A. Muller, Phys. Rev. Lett. 52, 2289 (1984).

[23] N.A. Hegab, M.A. Afifi, H.E. Atyia, M.I. Ismael, Acta Phys. Pol. A 119, 416 (2011)

[24] M. Barsoum, Fundamentals of Ceramics, Mc Graw-Hill, New York 1977,p. 543.

[25] W. Cao, R. Gerhardt, Solid State Ion. 42, 213 (1990).

[26] T.G. Abdel-Malak, M.E. Kassem, N.S. Aly, S.M. Kalil, Acta Phys. Pol. A 81, 675 (1992).

[27] R. Singh, R.P. Tandon, V.S. Panwar, S. Chandra, J. Appl. Phys. 69, 2504 (1991).

[28] T.M. Stevels, The Electrical Properties of Glasses. Handbuch in Phys., Springer, Berlin 1957, p. 350.

[29] H.W. Gibsen, R.J. Weagley, W.M. Prest, Jr. R. Mosher, S. Kaplan, J. Phys. Collo. C 6 (suppl.), 123 (1983).

[30] N.F. Mott, E.A. Davis, Electronic Processes in Non- Crystalline Materials, Clarendon Press, Oxford 1979.

[31] J.C. Giuntini, J.V. Zancheha, J. Non-Cryst. Solids 34, 57 (1979) 